\title{
Energy test of an efficient random laser emission collecting system
}

\author{
Ignacio Iparraguirre, ${ }^{\text {a,* Jon Azkargorta } \odot,{ }^{a} \text { Joaquin Fernández, }}$ \\ Sara García-Revilla $\odot,{ }^{a}$ and Rolindes Balda ${ }^{a, b, c}$ \\ ${ }^{a}$ Universidad del País Vasco UPV/EHU, Dpto. de Física Aplicada I, \\ Escuela de Ingeniería de Bilbao, Bilbao, Spain \\ ${ }^{b}$ Donostia International Physics Center DIPC, San Sebastián, Spain \\ ${ }^{\mathrm{c}}$ Materials Physics Center CSIC-UPV/EHU, San Sebastián, Spain
}

\begin{abstract}
The problem of light collection in random lasers (RLs) is addressed. As the radiation emitted by this system is Lambertian due to its spatial incoherence, a device based on an ellipsoidal revolution mirror is designed, developed, and tested in order to optimize the harvesting of the radiation emitted by the RL. The system provides a simple injection procedure of the emitted energy at the entrance of a multimode optical fiber. The results obtained show that the device has a net energy efficiency of 35\%, close to the theoretically expected one. (C) The Authors. Published by SPIE under a Creative Commons Attribution 4.0 Unported License. Distribution or reproduction of this work in whole or in part requires full attribution of the original publication, including its DOI. [DOI: 10 .1117/1.OE.60.1.010502]
\end{abstract}

Keywords: optics; light; lasers; scattering.

Paper 20201173L received Oct. 6, 2020; accepted for publication Jan. 15, 2021; published online Jan. 29, 2021.

\section{Introduction}

Random lasers (RLs) are attractive radiation sources for several applications, such as optical sensors, high-definition speckle-free imaging, lithography, holographic laser displays, etc. ${ }^{1-3}$ Many materials, such as colloidal dye solutions, solid-state dyes, polymers, crystal powders, etc., have been tested as RL sources, ${ }^{4-9}$ showing very varied energy efficiencies, pulse widths, and emission spectra ranges.

In particular, RLs based on $\mathrm{Nd}^{3+}$ ions have been tested as sources for speckle-free imaging in near-infrared and nanosecond pulse regime. ${ }^{10}$ The energy efficiency of all these systems is evidently given by the physical properties of the active medium itself and the pumping conditions, but the ability to capture the energy emitted by the RL, which is a very important issue to implement applications, has not been thoroughly discussed. The main trouble comes from the omnidirectionality of the emission. It is worth noting that the radiation emitted by an RL is Lambertian due to the very low transverse coherence of the emission. ${ }^{1,2}$

The aim of this work is to design, develop, and test a device that captures as much radiation as possible emitted in all directions by the RL. For this purpose, an ellipsoidal revolution mirror is employed. The external surface of the powdered material (laser active medium) is placed and centered on a focus of the ellipsoidal mirror, whereas on the other one, the optical head of a multimode optical fiber is located to collect the emitted laser radiation once the pumping radiation is eliminated by filtering. The device has been tested and its efficiency measured, turning out to be very close to our theoretical prediction.

\section{Design and Manufacturing of the Collecting Device}

The scheme of the device is shown in Fig. 1. The pumping beam is addressed to the mirror hole by means of an orientable mirror (a), and it is concentrated on the active medium by a mobile lens (b) to achieve the desired pumping size on its surface. The active medium consists of

*Address all correspondence to Ignacio Iparraguirre, i.iparraguirre@ehu.eus 


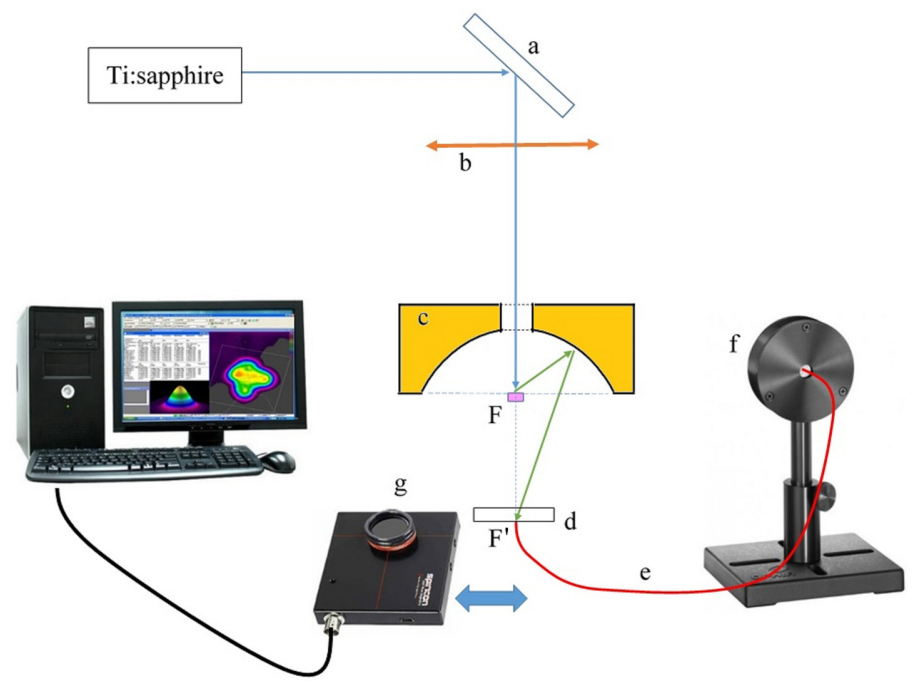

Fig. 1 Pumping and laser radiation collection system scheme: (a) orientable folder mirror, (b) mobile lens, (c) ellipsoidal mirror and active material just at focus $F$, (d) filter for pumping removal, (e) optical fiber with its head (with XYZ adjustment) just at focus $F^{\prime}$, (f) absolute energy meter, and (g) CCD camera.

a quartz cell $6 \mathrm{~mm}$ in diameter and about $2 \mathrm{~mm}$ deep. The cell is filled with powder to the limit of the cell walls so that the emitted radiation has no obstacle to reach the reflective surface of the ellipsoid. It is attached to the ellipsoidal mirror (c) in a fixed position by a thin rod. To facilitate focusing operations, the ellipsoidal mirror has been designed so that the focus $\mathrm{F}$ is in the truncating plane of the ellipsoid, as can be seen in Fig. 1. The outer surface of the powder is placed and centered on F. A fine adjustment of the orientation of the mirror (a) is required to achieve the pump beam just on $\mathrm{F}$, which is important to minimize geometric aberrations. Thus, within this simple scheme, if the pumping beam falls on $\mathrm{F}$, all the radiation emitted is directed after reflection to the other focus of the ellipsoid $\mathrm{F}^{\prime}$ where a filter (d) to remove the pumping radiation is placed. The head of a multimode optical fiber (e) with XYZ adjustment captures the radiation and carries it to an absolute energy meter (f) Ophir PE9. Eventually, a charge-coupled device (CCD) camera (g) (Spiricon SP503U from Ophir) is placed on $\mathrm{F}^{\prime}$ to analyze the beam quality. The distance between both foci of the ellipsoid is $40 \mathrm{~mm}$ and the periapsidal distance is $20 \mathrm{~mm}$, so the diameter of the mirror is $60 \mathrm{~mm}$. The diameter of the internal hole through which the pumping radiation passes is $10 \mathrm{~mm}$. It should be borne in mind that this concave mirror, as a paraxial element, gives an image at $\mathrm{F}^{\prime}(-60 \mathrm{~mm})$ of an object placed at $\mathrm{F}(-20 \mathrm{~mm})$. Therefore, its focal distance is $-15 \mathrm{~mm}$, and the paraxial lateral magnification of the mirror in our working conditions is -3 .

The specific parameters we mentioned above led us to reach a compromise between the maximum image size in $\mathrm{F}^{\prime}$ and the numerical aperture (NA) of the optical fiber. The minimum diameter (at 1/e) of the emission circle that we can get is close to $300 \mu \mathrm{m},{ }^{11,12}$ which becomes about $1 \mathrm{~mm}$ at the fiber head $\mathrm{F}^{\prime}$. Taking into account that the relationship between the incident energy within the fiber area and the total incident energy is given by $1-\exp \left[-\left(r_{f} / r_{o}\right)^{2}\right]$, where $r_{f}$ is the core radius of the fiber and $r_{o}$ is the beam radius at $1 / \mathrm{e}$. Thus, for a fiber of $1.5 \mathrm{~mm}$ in diameter and a beam of $1 \mathrm{~mm}$, coupling losses of approximately $10 \%$ are obtained. On the other hand, the NA of the fiber is 0.5 , which means an admittance half angle of approximately $30 \mathrm{deg}$, so the maximum angle of emission of the RL with respect to the semi-major axis of the ellipse is approximately $77 \mathrm{deg}$. The geometric losses due to the pumping hole or to the outermost area of the mirror are given by the factor $\cos ^{2} \theta_{1}-\cos ^{2} \theta_{2}$, with angles of $14 \mathrm{deg}$ (pumping hole) and $75 \mathrm{deg}$ (outermost area), which gives a loss close to $15 \%$. As an increase of the lateral augmentation would include more radiation within the NA of the fiber, whereas its decrease would produce the opposite effect, a compromise about concrete dimensions and distances was reached in order to have minimal coupling losses. Another way would be to make the mirror diameter smaller, but in addition to some new losses, it generates other mechanical inconveniences. On the 


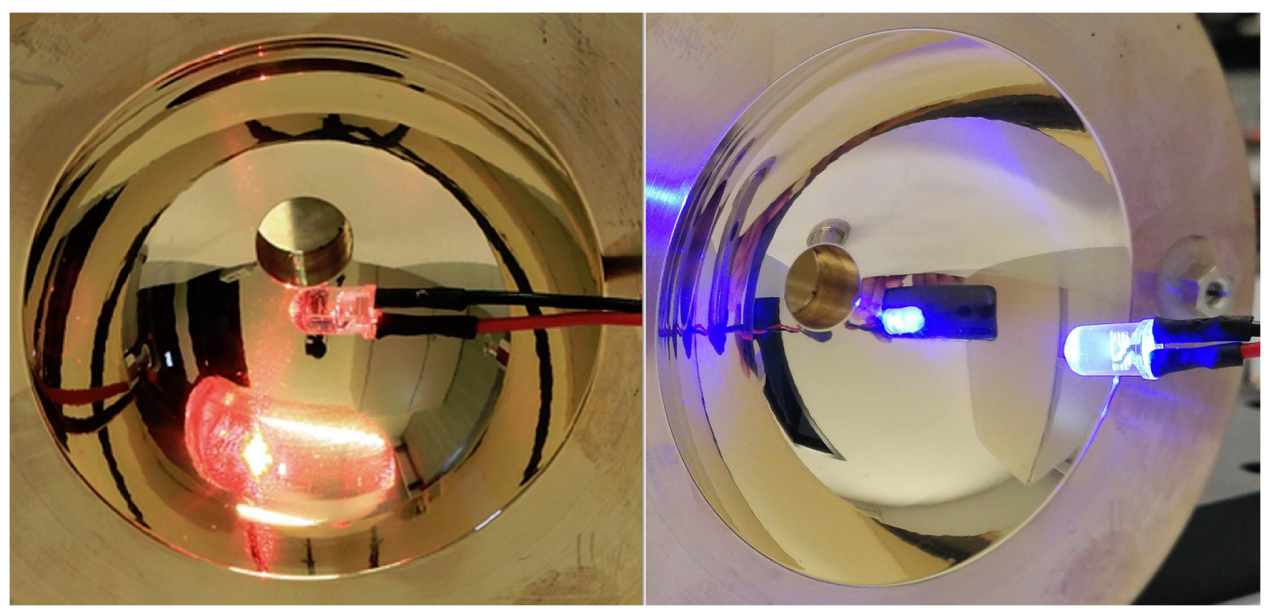

Fig. 2 Images of a visible LED from ellipsoidal mirror. Red, LED in F, image in $\mathrm{F}^{\prime}$; lateral augmentation -3 . Blue, LED in $\mathrm{F}^{\prime}$, image in $\mathrm{F}$; lateral augmentation $-1 / 3$.

other hand, it should be noted that the pumping areas we must use (about $0.1 \mathrm{~mm}^{2}$ ) limit the pumping energy to not much more than $2 \mathrm{~mJ}$, depending on the material, to avoid damage. ${ }^{11}$

As such a mirror is not commercially available, we designed it and contracted its mechanization. The chosen material was brass due to its high reflectivity and favorable properties for machining and polishing. Once machined, we polished it by using $0.06-\mu \mathrm{m}$ size powder (Eposil M11 from ATM) until the required optical quality was achieved. The optical test of the uncoated mirror, performed by measuring the reflection of a He-Ne laser beam, confirms a suitable optical quality. Likewise, we observed the image given by a light-emitting diode (LED) source at its foci positions to verify that the design is fulfilled and to qualitatively detect geometric aberrations, as well as the paraxial focal point lying $5 \mathrm{~mm}$ away from F. As a curiosity, Fig. 2 shows the images obtained with an LED source set at F (red) and with the LED set at $\mathrm{F}^{\prime}$ (blue).

To estimate the net yield, all the losses should be taken into account. First, those on the reflective uncoated brass surface of the ellipsoid. We have measured its absorption and diffusion losses around $1064 \mathrm{~nm}$, which give a value of about $15 \%$ (yield $\eta=0.85$ ). Second, the purely geometric losses due to the inlet port of the pumping and the limit of the fiber NA $(\eta=0.85)$. Third, losses due to the cell clamping system, which partially obstructs the radiation reflected by the mirror $(\eta=0.90)$. Fourth, beam-fiber geometrical coupling yield $(\eta=0.90)$. Finally, losses due to pump removal filter and fiber input and output $(\eta=0.80)$. From these partial yields, a net yield of about $45 \%$ is obtained.

\section{Test of the Collecting System}

To test the device, we have first obtained images of the focused beam for various powdered materials locating the $\mathrm{CCD}$ camera just at $\mathrm{F}^{\prime}$. Our aim was to observe the quality and size of the focused beam and the possible effects due to the geometric aberrations of the mirror. Figure 3 shows two images obtained with two different materials. In Fig. 3(a), we present the image on $\mathrm{F}^{\prime}$ of the radiation emitted by a nearly stoichiometric powdered material, $\mathrm{Nd}_{0.9} \mathrm{Y}_{0.1} \mathrm{Al}_{3}\left(\mathrm{BO}_{3}\right)_{4}$, pumped at $810 \mathrm{~nm}$ and with emission at $1063 \mathrm{~nm}$. In these powders, the diffusive absorption length is very short (about $10 \mu \mathrm{m}$ ), ${ }^{11-13}$ which explains the well-concentrated image obtained at $\mathrm{F}^{\prime}$, with a diameter at 1/e slightly smaller than $1 \mathrm{~mm}$. Figure $3(\mathrm{~b})$ shows the image on $\mathrm{F}^{\prime}$ of the radiation emitted by a ceramized oxisulfide, $\mathrm{La}_{2} \mathrm{O}_{2} \mathrm{~S}: \mathrm{Nd} 9 \%$, pumped at $819 \mathrm{~nm}$ and with emission at $1076 \mathrm{~nm}$ whose diffusive absorption length is very long compared to the first case. As a consequence, we find a much wider focused beam and a much more widespread radiation. It is worth noting that this difference is only due to the diffusive properties of the active medium, because the pump beam size is the same in both cases.

The absolute output energy of both powdered crystals have been directly measured with the energy meter by using our collecting device. The incident pump energy is measured 

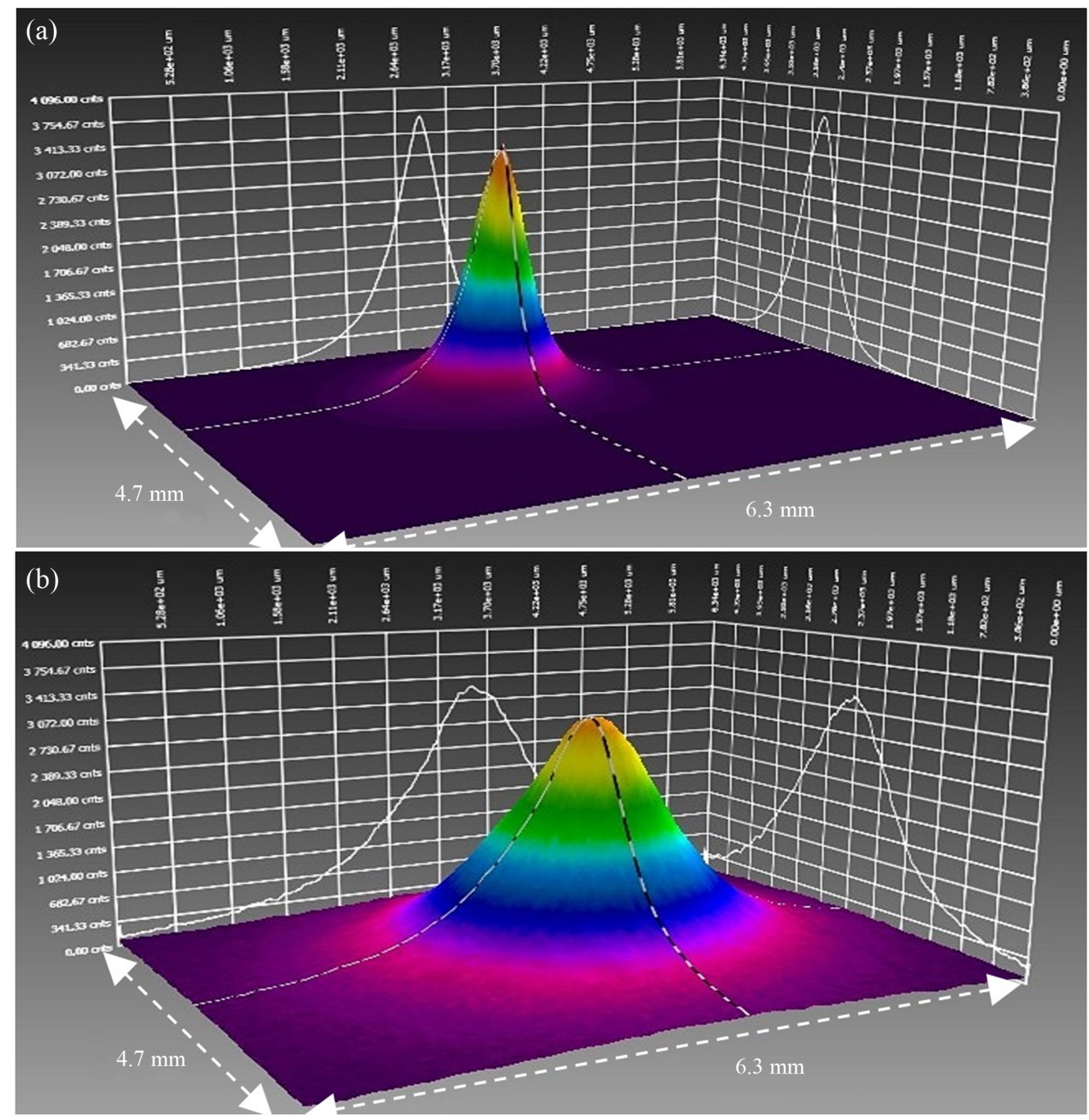

Fig. $3 \mathrm{CCD}$ images of the optimal focused beam. (a) $\mathrm{Nd}_{0.9} \mathrm{Y}_{0.1} \mathrm{Al}_{3}\left(\mathrm{BO}_{3}\right)_{4}$, pumped at 810-nm wavelength, emission at $1063 \mathrm{~nm}$. (b) Ceramics oxisulfide $\mathrm{La}_{2} \mathrm{O}_{2} \mathrm{~S}: \mathrm{Nd} 9 \%$, pumped at 819-nm wavelength, emission at $1076 \mathrm{~nm}$.

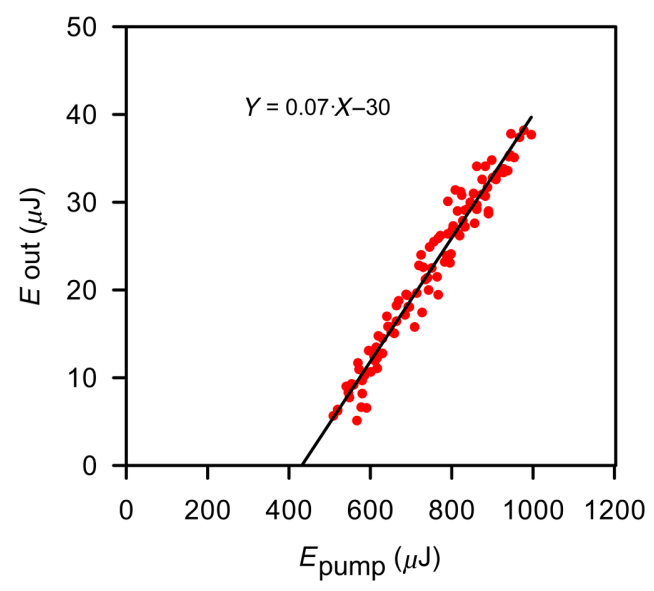

Fig. 4 Output energy (red points) and laser slope line of stimulated emission of the $\mathrm{Nd}_{0.9} \mathrm{Y}_{0.1} \mathrm{Al}_{3}\left(\mathrm{BO}_{3}\right)_{4}$ powder sample measured as function of the pump energy with the collecting system used after excitation at $810-\mathrm{nm}$ wavelength. 
simultaneously using the diffusion from a folder mirror with a fixed photodiode (Newport 818BB-21), previously calibrated by locating the energy meter above the pumping entrance on the ellipsoidal mirror. ${ }^{11-13}$ To get the device performance, the obtained laser slopes have been compared with their previously obtained ${ }^{12-14}$ total absolute slopes, and they are about $20 \%$. In the case of the ceramized oxisulfide, the absolute slope obtained is less than $1 \%$, showing a lousy beam-fiber coupling. For the near stoichiometric borate, the result is shown in Fig. 4. An absolute slope of $7 \%$ has been obtained. It is worth noting that if the efficiency of the collecting system was 1 , the slope would be 0.20 . Therefore, the energetic yield of the device in our working conditions is $35 \%$, which is close to our estimation from Sec. 2.

\section{Summary and Conclusions}

In this work, a device to collect as much energy as possible emitted from an RL has been designed, developed, and tested. The device is based on an ellipsoidal revolution mirror whose geometry is most suitable to capture the emitted radiation due to its Lambertian profile.

Images of the focused beam have been obtained for different active materials in order to observe the focus quality and the effect of geometric aberrations. These measurements reveal that active materials with the shortest possible diffusive absorption length are desired. Therefore, highly absorbent and diffusive materials, that is, stoichiometric (or almost) powders, are the most suitable ones.

The energy collecting efficiency of our system is $35 \%$, which is close to the previous theoretical estimation. This performance could be improved by applying a protected gold coating on the mirror surface, which would allow to reach a net yield not far from $50 \%$. An alternative option could be to have a more concentrated pump beam in order to improve the coupling between the focused beam and fiber head. Unfortunately, the minimum diameter (at 1/e) of the emission circle that we can get is close to $300 \mu \mathrm{m}$, which becomes about $1 \mathrm{~mm}$ at the fiber head $\left(\mathrm{F}^{\prime}\right)$.

The device is valid for use with other RLs, such as dyes RLs, and even to collect any Lambertian or similar emission type from a not too extensive source.

\section{Acknowledgments}

This work was supported by the Basque Government PIBA2018-24, Spanish Government MINECO under Project No. MAT2017-87035-C2-2-P (AEI/FEDER, UE), and Basque Country University (UPV/EHU) PPG17/07 and GIU17/014. We wish to thank J. E. Ruiz, researcher of the Laser Material Processing Group of the UPV/EHU, and J. M. Larrañaga, laboratory specialist technician (Mechanical Engineering Department), for their help in the design and polish of ellipsoidal mirrors, and R. Hueso and T. del Río of Planetary Science Group of the UPV/EHU for the photographs of the mirror images. The authors declare no conflicts of interest.

\section{References}

1. M. A. Noginov, Solid-State Random Lasers, Springer, Berlin (2005).

2. C. Gouedard et al., "Generation of spatially incoherent short pulses in laser pumped neodymium stoichiometric crystals and powders," J. Opt. Soc. Am. B 10(12), 2358-2363 (1993).

3. B. Redding, M. A. Choma, and H. Cao, "Speckle-free laser imaging using random laser illumination," Nat. Photonics 6, 355-359 (2012).

4. S. K. Cohen et al., "Random lasing in low molecular weight organic thin films," Appl. Phys. Lett. 99, 041114 (2011).

5. A. Consoli et al., "Large area resonant feedback random lasers based on dye-doped biopolymer films," Opt. Express 23, 29954-29963 (2015).

6. F. Yao et al., "Behaviors of random laser dye-doped nematic liquid crystals," Opt. Commun. 359, 15-19 (2016). 
7. M. Sobczyk and D. Szymanski, "Luminescence properties of $\mathrm{Nd}^{3+}$-doped $\mathrm{Y}_{2} \mathrm{Te}_{4} \mathrm{O}_{11}$ microcrystalline powder," J. Lumin. 183, 226-232 (2017).

8. J. Azkargorta et al., "Random laser action in Nd:YAG crystal powder," Materials 9, 369 (2016).

9. S. H. Lin et al., "Manipulation of polarized random lasers from dye-doped twisted nematic liquid crystals within wedge cells," IEEE Photonics J. 9, 1502208 (2017).

10. M. Barredo-Zuriarrain et al., "Speckle-free near-infrared imaging using a $\mathrm{Nd}^{3+}$ random laser," Laser Phys. Lett. 14, 106201 (2017).

11. J. Azkargorta et al., "Effects of pumping wavelength and pump density on the random laser performance of stoichiometric Nd crystal powders," Opt. Express 22, 27365-27372 (2014).

12. J. Azkargorta et al., "Random Laser properties of $\mathrm{Nd}^{3+}$ crystal powders," Opt. Express 26, 11787-11803 (2018).

13. I. Iparraguirre et al., "Laser action in $\mathrm{Nd}^{3+}$-doped lanthanum oxysulfide powders," Opt. Express 20, 23690-23699 (2012).

14. S. García-Revilla et al., "Random laser performance of $\mathrm{Nd}_{x} \mathrm{Y}_{1-x} \mathrm{Al}_{3}\left(\mathrm{BO}_{3}\right)_{4}$ laser cristal powders," Opt. Mater. 34(2) 461-464 (2011). 